\title{
UNIVERSITY OF LUND RADIOCARBON DATES II
}

\author{
SÖREN HÅKANSSON
}

Radiocarbon Dating Laboratory, Department of Quaternary Geology

University of Lund, Sweden

\section{INTRODUCTION}

The $\mathrm{C}^{14}$ measurements reported here were made in this laboratory between September 1967 and October 1968.

The measuring technique and equipment, and the treatment of samples were the same as reported previously (Radiocarbon, 1968, v. 10, p. 36-37). Age calculations are based on a contemporary value equal to 0.950 of the activity of the NBS oxalic acid standard and on a half-life for $\mathrm{C}^{14}$ of $5568 \mathrm{yr}$. Results are reported in years before 1950 (years B.P.), and in the A.D./B.C. scale. Errors quoted $( \pm 1 \sigma)$ include the standard deviations of the count rates for the unknown sample, the contemporary standard, and the background. Calculated errors smaller than 100 yr have been increased by rounding to that figure to take some account of the de Vries effect and the possible error in $\delta \mathrm{C}^{13}$. Corrections for deviations from the normal $\mathrm{C}^{13} / \mathrm{C}^{12}$ ratio $\left(\delta \mathrm{C}^{13}=-25.0 \%\right.$ in the P.D.B. scale) are applied for all samples. $\delta \mathrm{C}^{13}$ values quoted are relative to the P.D.B. standard.

The description of each sample is based on information provided by the person submitting the sample to the laboratory.

\section{ACKNOWLEDGMENTS}

The author thanks Mrs. Kerstin Lundahl who is responsible for sample preparations and routine operation of the dating equipment, and $\mathrm{R}$. Ryhage and his staff at the mass-spectrometric laboratory of Karolinska Inst., Stockholm, for making the $\mathrm{C}^{13}$ analyses. Special thanks are due Christian Cavallin for assistance with the electronic equipment.

I am also indebted to Bengt Hubendick, Naturhistoriska Mus., Göteborg, and Ake Hillefors, Dept. of Geog., Lund, for supplying contemporary shell samples.

\section{SAMPLE DESGRIPTIONS}

\section{GEOLOGIC SAMPLES}

\section{A. Sweden}

\section{Lu-85. Lagaoset}

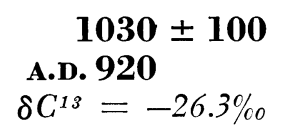

Sandy peat from river bank in mouth of Lagan R., Halland $\left(56^{\circ}\right.$ 33' N Lat, $12^{\circ} 57^{\prime}$ E Long). Coll. 1967 and subm. by Harald Svensson, Sci. Res. Council, Stockholm. Comments (H.S.): sample dated as part of investigation of former outflow of Lagan R. (S.H): $\mathrm{HCl}$ and $\mathrm{NaOH}$ pretreatment. 
Lu-102. Segevången 3

$6920 \pm 100$

4970 в.c.

$\delta C^{13}=-26.9 \%$

Small pieces of wood from Alnus stub in brushwood peat at Segevången, $4 \mathrm{~km} \mathrm{NE}$ of Malmö, Scania (55 $37^{\prime} \mathrm{N}$ Lat, $13^{\circ} 04^{\prime} \mathrm{E}$ Long). Peat layer rested on muddy clay, ca. $1.5 \mathrm{~m}$ above sea level, and was overlain by mud. Owing to selective pollen destruction in part of peat layer, pollen-analytical dating is unreliable (Welinder, 1968). Coll. 1967 and subm. by Stig Welinder, Dept. Quaternary Geol., Univ. of Lund. Comment: $\mathrm{HCl}$ and $\mathrm{NaOH}$ pretreatment.

$\begin{array}{lr}\text { Lu-104. Dösebacka } & \mathbf{2 4 , 0 2 0} \\ & \mathbf{2 2 , 0 7 0 ~ B . C . ~} \\ & \\ \text { Silty clay from intermoraine sediments at Dösebacka, } 3 \mathrm{~km} \mathrm{NNE} \text { of } & =-29.3 \% \\ \text { Kungälv, Bohuslän }\left(57^{\circ} 54^{\prime} \text { N Lat, } 11^{\circ} 54^{\prime} \text { E Long). Dösebacka area is }\right. \\ \text { described by Alin and Sandegren (1947) and Hillefors (1961). Coll. } 1967 \\ \text { and subm. by Ake Hillefors, Dept. Geog., Univ. of Lund. Comment: } \\ \text { only HCl pretreatment. Sample undersized; diluted with about same } \\ \text { amount "dead" } \mathrm{CO}_{2} \text { from anthracite coal. Date based on 10 1-day counts. }\end{array}$

\section{Ranviken Bay series}

Sediment samples from Ranviken Bay of Lake Immeln, ca. $30 \mathrm{~km}$ $\mathrm{N}$ of town of Kristianstad, NE Scania (56 $16^{\circ} \mathrm{N}$ Lat, $14^{\circ} 18^{\prime} \mathrm{E}$ Long). Coll. 1966-1967 and subm. by Gunnar Digerfeldt, Dept. Quaternary Geol., Univ. of Lund. Datings from Ranviken Bay were carried out in connection with an investigation of postglacial development of the lake and vegetation history of surrounding region (Digerfeldt, 1966). Samples come partly from main profile taken in central and deepest part of bay, partly from complementary profile taken in shallow water closer to shore (Livingstone sampler, diam. $60 \mathrm{~mm}$ ). Dated samples represent mostly pollen-zone boundaries and characteristic horizons in pollen sequence. Some samples are dated to obtain more accurate determination of rate of sediment deposition during different periods of lake development. Water depth at main profile $110 \mathrm{~cm}$, at complementary profile $90 \mathrm{~cm}$. Depths given in sample titles are below lake water level. All samples pretreated with $\mathrm{HCl}$.

Ranviken Bay, main profile:

\section{Lu-121. Ranviken, 765.0 to $770.0 \mathrm{~cm}$}

$$
\begin{aligned}
& 9850 \pm 100 \\
& 7900 \text { B.C. } \\
& \delta C^{13}=-27.3 \% o
\end{aligned}
$$

Detritus gyttja. At Pre-Boreal Betula maximum. Comment: date based on 3 1-day counts. 
Lu-122. Ranviken, 747.5 to $752.5 \mathrm{~cm}$

$9450 \pm 100$

7500 B.C.

$\delta C^{13}=-34.2 \%$

Detritus gyttja. Just below rational Corylus limit. Comment: date based on 3 1-day counts.

Lu-123. Ranviken, 742.5 to $747.5 \mathrm{~cm}$

$\mathbf{1 1 1 0}$

7360 B.c.

$\delta C^{13}=-31.1 \%$

Detritus gyttja. Just above rational Corylus limit.

Lu-117. Ranviken, 714.0 to $718.0 \mathrm{~cm}$

$8750 \pm 100$

6800 B.C.

Detritus gyttja. At rational Alnus limit.

Lu-118. Ranviken, 709.0 to $714.0 \mathrm{~cm}$

6620 B.C.

$\delta C^{13}=-31.3 \%$

Detritus gyttja. Just above rational Alnus limit.

Lu-119. Ranviken, 680.0 to $685.0 \mathrm{~cm}$

$8010 \pm 100$

6060 B.C.

$\delta C^{13}=-30.2 \%$

Detritus gyttja. At empirical Tilia limit.

Lu-120. Ranviken, 672.5 to $677.5 \mathrm{~cm}$

Detritus gyttja. Just above empirical Tilia limit.

Lu-193. Ranviken, 640.0 to $645.0 \mathrm{~cm}$

Detritus gyttja. Distinct increase of Quercus.

Lu-124. Ranviken, 602.5 to $607.5 \mathrm{~cm}$

$7790 \pm 100$

5840 в.C.

$\delta C^{13}=-31.2 \%$

$7390 \pm 100$

$\mathbf{5 4 4 0}$ в.C.

$\delta C^{13}=-30.5 \%$

$6980 \pm 100$

5030 B.c.

Detritus gyttja. Distinct further increase of Quercus. $\delta C^{13}=-30.5 \%$

Lu-194. Ranviken, 545.0 to $550.0 \mathrm{~cm}$

Detritus gyttja.

Lu-125. Ranviken, 487.5 to $492.5 \mathrm{~cm}$

$6140 \pm 100$

4190 в.c.

$\delta C^{13}=-30.4 \%$

$5840 \pm 100$

3890 B.C.

Detritus gyttja. Beginning decrease of Ulmus.

Lu-126. Ranviken, 467.5 to $472.5 \mathrm{~cm}$ $\delta C^{13}=-31.1 \%$

$5420 \pm 100$

3470 B.C.

Detritus gyttja. Ending decrease of Ulmus. 
Lu-195. Ranviken, 420.0 to $425.0 \mathrm{~cm}$

Detritus gyttja. Just above further increase of Quercus.

Lu-196. Ranviken, 350.0 to $355.0 \mathrm{~cm}$

Detritus gyttja.

Lu-127. Ranviken, 295.0 to $300.0 \mathrm{~cm}$

Detritus gyttja. At empirical Fagus limit.

Lu-128. Ranviken, 262.5 to $267.5 \mathrm{~cm}$

Detritus gyttja.

Lu-215. Ranviken, 177.5 to $182.5 \mathrm{~cm}$

Detritus gyttja. Slight further increase of Fagus.

Lu-197. Ranviken, 150.0 to $155.0 \mathrm{~cm}$

Detritus gyttja. Just below rational Fagus limit.

Lu-198. Ranviken, 135.0 to $140.0 \mathrm{~cm}$

Detritus gyttja. Just above rational Fagus limit.

Ranviken Bay, complementary profile:

Lu-129. Ranviken, 192.5 to $197.5 \mathrm{~cm}$

Detritus gyttja. Just below rational Fagus limit.

Lu-130. Ranviken, 182.5 to $187.5 \mathrm{~cm}$

Detritus gyttja. Just above rational Fagus limit.

Lu-131. Ranviken, 160.0 to $165.0 \mathrm{~cm}$

Detritus gyttja. Distinct further increase of Fagus.

Lu-132. Ranviken, 137.5 to $142.5 \mathrm{~cm}$
$4130 \pm 100$

2180 B.c.

$\delta C^{13}=-31.6 \%$

$3450 \pm 100$

1500 B.C.

$\delta C^{13}=-30.8 \%$

$2950 \pm 100$

1000 B.c.

$\delta C^{13}=-30.4 \%$ o

$2310 \pm 100$

360 B.C.

$\delta C^{13}=-30.7 \%$

$1880 \pm 100$

A.D. 70

$\delta C^{13}=-30.7 \%$

$1260 \pm 100$ A.D. 690

$\delta C^{13}=-31.6 \%$

$$
\begin{array}{r}
2300 \pm 100 \\
350 \text { B.C. } \\
\delta C^{13}=-29.5 \%
\end{array}
$$

$1920 \pm 100$

$$
\text { A.D. } 30
$$

$\delta C^{1 s}=-29.9 \%$

$1500 \pm 100$ A.D. 450

$\delta C^{13}=-31.1 \%$

A.D. 1040

$910 \pm 100$

$\delta C^{13}=-29.9 \%$

Detritus gyttja. Distinct decrease of Carpinus; culmination of Fagus. 


\section{Lu-133. Ranviken, 112.5 to $117.5 \mathrm{~cm}$}

Detritus gyttja. Just below rational Picea limit.

\section{Trummen series}

Continued from Lund I (Radiocarbon, 1968, v. 10, p. 40-43). Sediment samples from Lake Trummen, near city of Växjö, central S Sweden $\left(56^{\circ} 52^{\prime} \mathrm{N}\right.$ Lat, $14^{\circ} 50^{\prime} \mathrm{E}$ Long). Coll. 1966 and subm. by Gunnar Digerfeldt. Pollen zones according to Nilsson (1964). Water depth $150 \mathrm{~cm}$ at sampling point. Depths given are below lake water level. HCl pretreatment of all samples.

Lu-189. Trummen, 530.0 to $535.0 \mathrm{~cm}$

$8940 \pm 100$ 6990 B.C.

Detritus gyttja. Beginning decrease of Pinus in Pollen Zone BO 1.

Lu-190. Trummen, 460.0 to $465.0 \mathrm{~cm}$

$8060 \pm 100$ 6110 B.C. $\delta C^{13}=-30.2 \%$

Detritus gyttja. Increase of Quercus in Pollen Zone BO 2.

Lu-191. Trummen, 395.0 to $400.0 \mathrm{~cm}$

$6180 \pm 100$

4230 B.C.

$\delta C^{13}=-30.5 \%$

Detritus gyttja. Increase of Quercus in Pollen Zone AT.

Lu-192. Trummen, 255.0 to $260.0 \mathrm{~cm}$

Detritus gyttja. Minimum of Quercus in Pollen Zone SB 2.

\section{Kolbengtserödssjön series, 1965}

Sediment samples from Lake Kolbengtserödssjön, Bohuslän $\left(58^{\circ} 15^{\prime}\right.$ N Lat, $11^{\circ} 57^{\prime}$ E Long). Stratigraphic sequence from top to bottom: 487 $\mathrm{cm}$ lacustrine gyttja, $52 \mathrm{~cm}$ marine clay gyttja, $24 \mathrm{~cm}$ lacustrine gyttja, $39 \mathrm{~cm}$ marine clay gyttja, $51 \mathrm{~cm}$ lacustrine gyttja, marine clay gyttja and clay. Samples taken with Livingstone sampler, diam. $36 \mathrm{~mm}$. Water depth ca. $7 \mathrm{~m}$ at sampling point. Depths given in sample descriptions are below lake water level. Coll. 1965 and subm. by Gösta Persson, Dept. Quaternary Geol., Univ. of Lund.

\section{Lu-136. Kolbengtserödssjön B3}

$6810 \pm 120$

4860 в.C.

$\delta C^{13}=-30.2 \%$

Lacustrine gyttja, 11.67 to $11.77 \mathrm{~m}$. Rational Tilia limit at $11.77 \mathrm{~m}$. Comment: date based on 3 l-clay counts. 


\title{
Lu-137. Kolbengtserödssjön B4
}

$6870 \pm 125$

4920 B.C.

$\delta C^{13}=-29.8 \%$

Lacustrine gyttja, 11.77 to $11.87 \mathrm{~m}$. Comment: date based on 3 1-day counts.

\section{Lu-141. Kolbengtserödssjön D4}

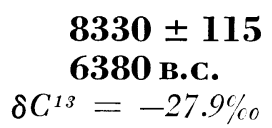

Lacustrine gyttja, 13.02 to $13.12 \mathrm{~m}$. Rational Quercus limit at 13.02 m. Comment: date based on 4 1-day counts.

\section{Lu-140. Kolbengtserödssjön D6}
$8400 \pm 105$
6450 B.c.
$\delta C^{13}=-25.1 \%$

Lacustrine gyttja, 13.22 to $13.32 \mathrm{~m}$. Dates rational Ulmus limit. Comment: date based on 3 1-day counts.

\section{Lu-139. Kolbengtserödssjön D8}

\author{
$8710 \pm 145$ \\ 6760 в.c. \\ $\delta C^{13}=-29.8 \%$
}

Lacustrine gyttja, 13.42 to $13.52 \mathrm{~m}$. Rational Alnus limit at 13.52 m. Comment: date based on 4 1-day counts. All samples undersized; diluted with "dead" $\mathrm{CO}_{2}$ from anthracite coal. $\mathrm{HCl}$ pretreatment. Two samples of marine clay gyttja were processed but did not yield enough $\mathrm{CO}_{2}$ for dating.

\section{Kolbengtserödssjön series, 1968}

Sediment samples from Lake Kolbengtserödssjön. Stratigraphic sequence from top to bottom: $252 \mathrm{~cm}$ lacustrine gyttja, $7 \mathrm{~cm}$ marine clay, $3 \mathrm{~cm}$ lacustrine gyttja, $2 \mathrm{~cm}$ marine sand, $100 \mathrm{~cm}$ lacustrine gyttja, 6 $\mathrm{cm}$ marine clay, $165 \mathrm{~cm}$ lacustrine gyttja, $5 \mathrm{~cm}$ marine clay, lacustrine gyttja. Water depth ca. $8 \mathrm{~m}$ at sampling point. Depths given in sample descriptions are below lake water level. Samples were taken with Livingstone sampler, diam. $36 \mathrm{~mm}$, ca. $300 \mathrm{~m}$ from sampling point for 1965 series. Coll. 1968 and subm. by Gösta Persson. HCl pretreatment.

\section{Lu-216. Kolbengtserödssjön 1}

$8020 \pm 100$

6070 в.C.

$$
\delta C^{13}=-29.8 \%
$$

Lacustrine gyttja, 11.22 to $11.37 \mathrm{~m}$. Dates rational Quercus limit.

\section{Lu-217. Kolbengtserödssjön 2}

$$
\begin{array}{r}
\mathbf{8 5 9 0} \pm 153 \\
\mathbf{6 6 4 0} \text { в.C. } \\
\delta C^{13}=-29.1 \%
\end{array}
$$

Lacustrine gyttja, 11.54 to $11.64 \mathrm{~m}$. Dates rational Alnus limit. Comment: sample undersized; diluted. 


\section{Lu-218. Kolbengtserödssjön 3}

Lacustrine gyttja just below marine clay, 13.40 to $13.50 \mathrm{~m}$. Dates Early Boreal marine transgression. Comment: sample undersized; diluted.

\section{Vassbosjön series}

Sediment samples from Lake Vassbosjön, Bohuslän $\left(58^{\circ} 16^{\prime} \mathrm{N}\right.$ Lat, $12^{\circ} 00^{\prime} \mathrm{E}$ Long). Stratigraphic sequence from top to bottom: $311 \mathrm{~cm}$ lacustrine gyttja, $2 \mathrm{~cm}$ marine clay, $95 \mathrm{~cm}$ lacustrine gyttja, marine clay. Samples taken with Livingstone sampler, diam. $36 \mathrm{~mm}$. Water depth ca. $4 \mathrm{~m}$ at sampling point. Depths given in sample descriptions are below lake water level. Coll. 1968 and subm. by Gösta Persson. HCl pretreatment.

\section{Lu-219. Vassbosjön 1}

Lacustrine gyttja, 7.85 to $7.95 \mathrm{~m}$. Rational Corylus limit at $7.95 \mathrm{~m}$.

\section{Lu-220. Vassbosjön 2}

$9700 \pm 111$

$\mathbf{7 7 5 0}$ B.C.

Lacustrine gyttja, 7.95 to $8.05 \mathrm{~m}$.

$$
\delta C^{13}=-26.9 \%
$$

\section{Ryssjön series}

Sediment samples from Lake Ryssjön situated at coast of central Blekinge, SE Sweden $\left(56^{\circ} 10^{\prime} \mathrm{N}\right.$ Lat, $15^{\circ} 05^{\prime} \mathrm{E}$ Long). Alt $3 \mathrm{~m}$; size $300 \times 400 \mathrm{~m}$; max. depth $2.3 \mathrm{~m}$; mean depth $1.7 \mathrm{~m}$. Lacustrine sediments are overlying marine gyttja. Investigated to date some horizons in pollen diagram. Samples were cut from core taken with Livingstone sampler (diam. $60 \mathrm{~mm}$ ). Water depth $170 \mathrm{~cm}$ at sampling point. Depths given in sample titles are below lake water level. Pollen zones according to Berglund (1966). Coll. 1967 and subm. by C. E. Nylander and B. E. Berglund, Dept. Quaternary Geol., Univ. of Lund. HCl pretreatment of all samples.

\section{Lu-187. Ryssjön 0, 360 to $365 \mathrm{~cm}$}

$4110 \pm 100$

2160 B.C.

$\delta C^{13}=-21.3 \%$

Brackish, clayey detritus gyttja. Just below isolation level in upper part of SB 1 .

Lu-147. Ryssjön 1, 342 to $347 \mathrm{~cm}$

$3370 \pm 100$

1420 B.C.

$\delta C^{13}=-31.1 \%$

Lacustrine detritus gyttja. Just above isolation level in lower part of SB 2. 


\section{Lu-154. Ryssjön 2, 337 to $342 \mathrm{~cm}$}

$3230 \pm 100$

1280 B.C.

Lacustrine detritus gyttja. Slightly above Ryssjön 1.

$$
\delta C^{13}=-30.1 \%
$$

Lu-148. Ryssjön 3, 304 to $309 \mathrm{~cm}$

$2860 \pm 100$

910 B.C.

Lacustrine detritus gyttja. Distinct increase of Betula

Lu-149. Ryssjön 4, 285 to $290 \mathrm{~cm}$

$2700 \pm 100$

750 в.C.

Lacustrine detritus gyttja. At empiric Fagus limit.

$$
\delta C^{13}=-28.6 \%
$$

Lu-150. Ryssjön 6, 268 to $273 \mathrm{~cm}$

$2430 \pm 100$

480 B.C.

$\delta C^{13}=-27.6 \%$

Lacustrine detritus gyttja. Level with increased cultural activity.

Lu-151. Ryssjön 7, 245 to $250 \mathrm{~cm}$

$1960 \pm 100$

10 B.C.

$\delta C^{13}=-28.2 \%$

Lacustrine detritus gyttja. Just below rational Fagus limit.

Lu-155. Ryssjön 8, 240 to $245 \mathrm{~cm}$

$1860 \pm 100$

A.D. 90

$\delta C^{13}=-32.2 \%$

Lacustrine detritus gyttja. Level with increasing Fagus values.

Lu-152. Ryssjön 9, 220 to $225 \mathrm{~cm}$

$1160 \pm 100$
A.D. 790
$\delta C^{13}=-30.1 \%$

Lacustrine detritus gyttja. Fagus culmination, rational limit of Juniperus and increased cultural activity.

Lu-153. Ryssjön 10, 205 to $210 \mathrm{~cm}$

Lacustrine detritus gyttja. Juniperus culmination.

Lu-188. Ryssjön 11, 187 to $192 \mathrm{~cm}$
A.D. 1120
$\delta C^{13}=-27.9 \%$

$830+100$

$610 \pm 100$

A.D. 1340

$\delta C^{13}=-28.6 \%$

Lacustrine detritus gyttja. Decrease of Juniperus and empiric limit of Picea.

\section{Högestad series}

Peat samples from spring mire at Högestad, ca. $11 \mathrm{~km}$ NNE of Ystad, Scania (55 $31^{\prime} \mathrm{N}$ Lat, $13^{\circ} 54^{\prime} \mathrm{E}$ Long). Site described by Kurck (1922). Coll. 1919 by C. Kurck; subm. by Tage Nilsson, Dept. Quaternary Geol. Univ. of Lund. 


\section{Lu-175. Högestad, Layer 2}

$9950 \pm 100$

8000 B.C.

$\delta C^{13}=-28.9 \%$

Highly humified Amblystegium peat, almost non-calcareous, from bottom of Layer 2. Comments (T.N.): Layer 2 dated by pollen analysis to transition between Scanian Pollen Zones PB and BO 1. (S.H.): HCl pretreatment.

\section{Lu-174. Högestad, Layer 4}

Slightly humified Amblystegium peat from Layer 4. Thin layers of highly calcareous mud (Swed. bleke) were intercalated in peat. Comments (T.N.): Layer 4 dated by pollen analysis to upper part of Scanian Pollen Zone BO 1. (S.H.): HCl pretreatment. Sample charred in nitrogen atmosphere prior to burning. Slightly undersized; diluted with "dead" $\mathrm{CO}_{2}$ from anthracite coal.

\section{Evensås series, fossil marine shells}

Shells from 2 shell beds at Evensås on island of Skaftölandet, Bohuslän $\left(58^{\circ} 16^{\prime} \mathrm{N}\right.$ Lat, $11^{\circ} 30^{\prime} \mathrm{E}$ Long). Shell beds described by De Geer (1910), Antevs (1917), and Nilsson (1927). Alt of shell bed Evensås I $35 \mathrm{~m}$; alt of shell bed Evansås III ca. $29 \mathrm{~m}$; distance between shell beds ca. $100 \mathrm{~m}$. Shell bed Evensås III is divided into lower and upper part, with faunas differing from each other and from fauna in Evensås I. Selected thick shells were dated. Coll. 1926 and subm. by Tage Nilsson.

\section{Lu-157. Evensås I, Littorina + Patella, $\mathrm{i}$}

$$
\begin{gathered}
9030 \pm 100 \\
\mathbf{7 0 8 0} \text { в.c. } \\
\delta C^{13}=+0.1 \%
\end{gathered}
$$

Littorina littorea, L. obtusata, and Patella vulgata from Evensås I. Comments (T.N.): Evensås I contains fairly homogeneous fauna without significant differences among different layers. (S.H.): inner $50 \%$ of shell thickness was used.

\section{Lu-158. Evensås I, Littorina + Patella, o}

$$
\begin{gathered}
\mathbf{9 0 4 0} \pm 110 \\
\mathbf{7 0 9 0} \text { в.C. } \\
\delta C^{13}=+2.2 \%
\end{gathered}
$$

Shell layer surrounding part used for Lu-157. Comment: layer corresponds to $34 \%$ of shells; $16 \%$ removed by acid washing. Sample undersized; diluted with "dead" $\mathrm{CO}_{2}$ from anthracite coal. Date based on 3 1-day counts.

\section{Lu-159. Evensås I, Saxicava arctica, i}

$$
\begin{gathered}
11,110 \pm 100 \\
9160 \text { в.C. } \\
\delta C^{13}=+0.9 \%
\end{gathered}
$$

Saxicava arctica from Evensås I. Comment: inner $47 \%$ of shell thickness was used. Date based on 3 1-day counts. 


\section{Lu-160. Evensås I, Saxicava arctica, o}

$10,960 \pm 115$

9010 в.C.

$\delta C^{13}=+0.9 \%$

Shell layer surrounding part used for Lu-159. Comment: layer corresponds to $31 \%$ of shells; $22 \%$ removed by acid washing.

\section{Lu-161. Evensås I, Mya truncata, i}

$$
\begin{gathered}
11,220 \pm 110 \\
9270 \text { в.c. } \\
\delta C^{13}=+0.9 \% o
\end{gathered}
$$

Mya truncata from Evensås I. Comment: inner $42 \%$ of shell thickness was used.

\section{Lu-162. Evensås I, Mya truncata, o}

$$
\begin{gathered}
10,970 \pm 105 \\
\text { 9020 в.c. } \\
\delta C^{13}=+0.5 \%
\end{gathered}
$$

Shell layer surrounding part used for Lu-161. Comment: layer corresponds to $33 \%$ of shells; $25 \%$ removed by acid washing.

\section{Lu-163. Evensås III, Pecten islandicus, Sample 1 \\ $11,460 \pm 115$ 9510 B.C. \\ $\delta C^{13}=+1.9 \%$}

One well-preserved shell of Pecten islandicus from presumably lower part of Evensås III. Comment: inner $68 \%$ of shell thickness was used.

\section{Lu-164. Evensås III, Pecten islandicus, Sample 2 \\ $12,330 \pm 125$ \\ 10,380 в.c. \\ $\delta C^{13}=+2.5 \%$}

One well-preserved shell of Pecten islandicus from presumably lower part of Evensås III. Comment: inner $90 \%$ of shell thickness was used. General Comments (T.N.): shell beds are now generally realized to be accumulations containing mixed, partly redeposited fauna. Especially the large Saxicava arctica, Mya truncata, and Pecten islandicus, considered to indicate arctic or subarctic environment, are thought to be redeposited. The first two are ca. $2000 \mathrm{yr}$ older than are Littorina and Patella from same section. (S.H.): corrections for deviations from normal $\mathrm{C}^{13} / \mathrm{C}^{12}$ ratio for terrestrial plants $\left(\delta C^{13}=-25.0 \%\right.$ in P.D.B. scale) are applied also for shell samples. No corrections are made for apparent age of sea water (cf. Lu-234 through Lu-236).

\section{Fjärås bräcka series, fossil shells}

Shells from $\mathrm{S}$ part of Fjärås bräcka, Fjärås parish, $10 \mathrm{~km} \mathrm{SE}$ of Kungsbacka, Halland (57 $27^{\prime} \mathrm{N}$ Lat, $12^{\circ} 11^{\prime}$ E Long). Layers with shells overlie glaciofluvial gravel and are overlain by laminated sand; alt ca. $56 \mathrm{~m}$. Coll. 1965 by Per Wedel; subm. by Tage Nilsson.

\section{Lu-165. Fjärås bräcka, Mytilus, i}

Mytilus shells from Fjärås bräcka. Comment: inner $25 \%$ of shell thickness was used. 


\section{Lu-166. Fjärås bräcka, Mytilus, o \\ $13,250 \pm 135$ \\ 11,300 в.c. \\ $\delta C^{13}=-0.3 \%$}

Shell layer surrounding part used for Lu-165. Comment: layer corresponds to $35 \%$ of shells; $40 \%$ removed by acid washing.

\section{Lu-167. Fjärås bräcka, Balanus, i \\ $12,850 \pm 130$ \\ 10,900 в.C. \\ $\delta C^{13}=-1.9 \%$}

Balanus shells from Fjärås bräcka. Comment: inner $25 \%$ of shell thickness was used.

\section{Lu-168. Fjärås bräcka, Balanus, o}

$12,620 \pm 130$

10,670 в.C.

$\delta C^{13}=-3.1 \%$

Shell layer surrounding part used for Lu-167. Comment: layer corresponds to $38 \%$ of shells; $37 \%$ removed by acid washing.

\section{Lu-169. Fjärås bräcka, Balanus, organic \\ $12,310 \pm 650$ \\ 10,360 B.c. \\ $\delta C^{13}=-19.7 \%$}

Balanus shells from Fjärås bräcka. Comment: organic fraction from ca. $1 \mathrm{~kg}$ shells was used; yielded ca. $13 \%$ of quantity needed for normal filling. Diluted with "dead" $\mathrm{CO}_{2}$ from anthracite coal. Date based on 3 1-day counts.

General Comments: corrections for deviations form normal $\mathrm{C}^{13} / \mathrm{C}^{12}$ ratio for terrestrial plants $\left(\delta C^{13}=-25.0 \%\right.$ in P.D.B. scale) are applied also for shell samples and organic shell fraction. No corrections are made for apparent age of sea water (cf. Lu-234 through Lu-236).

Lu-170. Hjälmared, Balanus + Mytilus, i

$$
\begin{gathered}
11,610 \pm 115 \\
9660 \text { B.c. } \\
\delta C^{13}=-3.1 \%
\end{gathered}
$$

Balanus and Mytilus shells from Hjälmared, $7 \mathrm{~km} \mathrm{NE}$ of Kungsbacka, Halland (57 $32^{\prime} \mathrm{N}$ Lat, $12^{\circ} 10^{\prime} \mathrm{E}$ Long). Stratigraphic sequence from bottom to top: glaciofluvial gravel, brown varved clay, bluish clay with shells, washed gravel; alt ca. $30 \mathrm{~m}$. Coll. 1968 by Per Wedel; subm. by Tage Nilsson. Comment: inner $23 \%$ of shell thickness was used.

\section{Lu-171. Hjälmared, Balanus + Mytilus, o}

$$
\begin{gathered}
11,470 \pm 110 \\
9520 \text { в.c. } \\
\delta C^{13}=-1.5 \%
\end{gathered}
$$

Shell layer surrounding part used for Lu-170. Comment: layer corresponds to $22 \%$ of shells; $55 \%$ removed by acid washing.

\section{Lu-200. Hjärtumåsen, Mya truncata, i}

$$
\begin{gathered}
11,590 \pm 110 \\
9640 \text { в.c. } \\
\delta C^{13}=+0.8 \% o
\end{gathered}
$$

Shells of Mya truncata from Hjärtumåsen, Hjärtum parish, Bohuslän $\left(58^{\circ} 11^{\prime} \mathrm{N}\right.$ Lat, $12^{\circ} 06^{\prime} \mathrm{E}$ Long). Shells were from undistinctly varved 
gray glacial marine clay, overlying glaciofluvial sand and gravel; alt ca. $80 \mathrm{~m}$. Ref. Hillefors (1968). Coll. 1963 and subm. by Åke Hillefors, Dept. Geog., Univ. of Lund. Comment: inner $35 \%$ of shell thickness was used.

\section{Lu-199. Hjärtumåsen, Mya truncata, o}

$$
11,540 \pm 100
$$

Shell layer surrounding part used for Lu-200. Comment: layer corresponds to $37 \%$ of shells; $28 \%$ removed by acid washing. Date based on 3 1-day counts.

\section{Contemporary marine shells series}

Lu-235. Rörö, Buccinum undatum Apparent Age $410 \pm 46$ $\delta C^{13}=-0.5 \%$

Buccinum undatum shells from Rörö, $\mathrm{N}$ archipelago of Göteborg $\left(57^{\circ} 47^{\prime} \mathrm{N}\right.$ Lat, $11^{\circ} 37^{\prime} \mathrm{E}$ Long). Coll. probably between 1920 and 1940; subm. by Bengt Hubendick, Naturhist. Mus., Göteborg. Comment: inner $72 \%$ of shell thickness was used.

\section{Lu-234. Rörö, Balanus}

Apparent Age $370 \pm 57$ $\delta C^{13}=-0.5 \% c$

Small Balanus shells found on Buccinum shells used for Lu-235. Comment: $100 \%$ of shells were used. Sample undersized; diluted.

\section{Lu-236. Härön, Mytilus}

Apparent Age $430 \pm 46$ $\delta C^{13}=+0.1 \%$

Mytilus shells from Härön, Bohuslän $\left(58^{\circ} 01^{\prime} \mathrm{N}\right.$ Lat, $11^{\circ} 31^{\prime} \mathrm{E}$ Long). Coll. 1968 and subm. by Åke Hillefors. Comment: inner $80 \%$ of shell thickness was used. Sample taken ca. $1 / 2 \mathrm{~m}$ below surface of accumulation of clam shells. Clams were used as fishing bait during period 1920 to 1950 . General Comment: corrections for deviations from normal $\mathrm{C}^{13} / \mathrm{C}^{12}$ ratio for terrestrial plants $\left(\delta C^{13}=-25.0 \%\right.$ in P.D.B. scale) are applied also for these shell samples. Unaugmented counting errors are given.

\footnotetext{
Abisko series

Sediment samples (Livingstone core sampler, diam. $36 \mathrm{~mm}$ ) from Lake Vuolep Njakajaure, near Abisko, Torne Lappmark, N Sweden $\left(68^{\circ} 20^{\prime} \mathrm{N}\right.$ Lat, $18^{\circ} 45^{\prime} \mathrm{E}$ Long). Alt $408 \mathrm{~m}$; size ca. $500 \times 600 \mathrm{~m}$. Samples taken from 2 profiles, one 75 to $100 \mathrm{~m}$ from SE shore (Gp 1, depth of water $4.45 \mathrm{~m}$ ), other approx. in middle of lake (Gp 3, depth $12.5 \mathrm{~m}$ ). Peat samples from mire near lake (Bp 100, 101). Coll. 1963 to 1966 and subm. by Mats Sonesson, Dept. of Plant Ecol., Univ. of Lund. Dating is part of current investigations on vegetational dynamics of Torneträsk area. Dated samples refer to boundaries of pollen zones described by Sonesson (1968). Depths given in sample titles are below water level and surface of mire respectively.
} 
Lu-176. Vuolep Njakajaure, Gp 1, 665 to $675 \mathrm{~cm}$

$9420 \pm 130$

$\mathbf{7 4 7 0}$ B.c.

$\delta C^{13}=-29.8 \%$

Detritus gyttja. From lower part of Pollen Zone $\mathrm{T} 1$ at mineral substratum. Low frequencies of Pinus, high of Betula, decreasing Hippophae and low frequencies of Juniperus. Comment: date based on 3 1day counts.

\section{Lu-205. Vuolep Njakajaure, Gp 1,} 660 to $665 \mathrm{~cm}$
$9240 \pm 180$ 7290 B.c.
$\delta C^{13}=-32.5 \%$

Detritus gyttja. From middle part of Zone T 1. Low frequencies of Pinus, high of Betula, increasing Juniperus. Comment: date based on 3 1-day counts.

$\begin{array}{llr}\text { Lu-177. } & \text { Vuolep Njakajaure, Gp 1, } & 6690 \pm 125 \\ 600 \text { to } 610 \mathrm{~cm} & \mathbf{4 7 4 0} \text {..c. } \\ & \delta C^{13}=-33.1 \%\end{array}$

Detritus gyttja. Distinctly increasing Pinus, decreasing Betula and Alnus, just below Zone Boundary T 2/T 3 .

$\begin{array}{llr}\text { Lu-178. } & \text { Vuolep Njakajaure, Gp 1, } & \mathbf{6 6 0 0} \pm 100 \\ 590 \text { to } 600 \mathrm{~cm} & \mathbf{4 6 5 0} \text { B.c. } \\ & \delta C^{13}=-33.8 \%\end{array}$

Detritus gyttja. Distinctly increasing Pinus and decreasing Betula, just above Zone Boundary 'T 2/T 3. Comment: date based on 3 1-day counts.

Lu-179. Vuolep Njakajaure, Gp 1, 505 to $515 \mathrm{~cm}$

$\mathbf{3 5 8 0} \pm 100$
1630 в.с.
$\delta C^{13}=-31.6 \%$

Detritus gyttja. Distinctly decreasing Pinus and increasing Betula, just below Zone Boundary $\mathrm{T}$ 3/T 4. Lu-180. Vuolep Njakajaure, Gp 1,
495 to $505 \mathrm{~cm}$
$3360 \pm 100$
1410 в.C.
$\delta C^{13}=-30.1 \%$

Detritus gyttja. Distinctly decreasing Pinus and increasing Bctula, just above Zone Boundary T $3 / \mathrm{T} 4$.

$\begin{array}{llr}\text { Lu-181. } & \text { Vuolep Njakajaure, Gp 1, } & 2370 \pm 100 \\ 472 \text { to } 482 \mathrm{~cm} & 420 \text { B.c. } \\ & & \delta C^{13}=-29.5 \%\end{array}$

Detritus gyttja. Increase of Pinus, decrease of Betula, at Sub-Zone Boundary $\mathrm{T} 4 \mathrm{a} / \mathrm{b}$.

$\begin{array}{llr}\text { Lu-201. } & \text { Vuolep Njakajaure, Gp 3, } & \mathbf{8 9 8 0} \pm 100 \\ 1550 \text { to } 1563 \mathrm{~cm} & \mathbf{7 0 3 0} \text {..c. } \\ & \delta C^{13}=-30.7 \%\end{array}$

Detritus gyttja. Below rational frequencies of Alnus at Zone Boundary $\mathrm{T} 1 / \mathrm{T} 2$. 


\section{Lu-206. Vuolep Njakajaure, Gp 3, 1495 to $1505 \mathrm{~cm}$}

Detritus gyttja. Distinctly increasing Pinus, decreasing Betula and Alnus, just below Zone Boundary $\mathrm{T} 2 / \mathrm{T}$ 3. Comment: only NaOHsoluble fraction used for dating.
Lu-202. Vuolep Njakajaure, Gp 3, 1485 to $1495 \mathrm{~cm}$
$6190 \pm 100$
4240 в.C.
$\delta C^{13}=-31.3 \%$

Detritus gyttja. Distinctly increasing Pinus and decreasing Betula, just above Zone Boundary T 2/T 3.

\section{Lu-203. Vuolep Njakajaure, Gp 3, $\quad 3380 \pm 100$ 1360 to $1370 \mathrm{~cm}$ \\ 1430 B.C. \\ $\delta C^{13}=-31.3 \%$}

Detritus gyttja. Distinctly decreasing Pinus and increasing Betula, just below Zone Boundary T 3/T 4 .

\section{Lu-204. Vuolep Njakajaure, Gp 3, 1325 to $1335 \mathrm{~cm}$}

$2410 \pm 100$

460 B.C.

Detritus gyttja. Decrease of Pinus, increase of Betula, just above Sub-Zone Boundary T 4a/b.

Lu-228. Abisko, Bp 100, 101; 285 to $298 \mathrm{~cm} \begin{gathered}\mathbf{6 3 3 0} \pm 100 \\ \begin{aligned} 4380 \text { B.c. } \\ \delta C^{13}=-26.8 \%\end{aligned}\end{gathered}$

Sphagnum peat. Increasing Pinus, decreasing Betula and Alnus, just above mineral substratum. Comment: date based on 3 1-day counts.

\section{Lu-229. Abisko, Bp 100, 101; 95 to $115 \mathrm{~cm}$ \\ $2470 \pm 100$ \\ 520 в.C. \\ $\delta C^{13}=-26.5 \%$}

Scorpidium peat. Distinctly decreasing Pinus and increasing Betula, at Zone Boundary T 3/T 4. Comment: date based on 3 1-day counts. Comment on treatment: all samples except Lu-201 and Lu-202 undersized; diluted with "dead" $\mathrm{CO}_{2}$ from anthracite coal. HCl pretreatment of all samples. Possibility of contamination with graphite was studied on material just below sample Lu-176 (Sonesson, 1968). In one case (Sample $\mathrm{Lu}-206) \mathrm{NaOH}$-soluble fraction was dated for same reason.

\section{Vassijaure series}

Peat samples coll. by digging from mire ca. $1 \mathrm{~km} \mathrm{~N}$ of Vassijaure R.R. Sta., Torne Lappmark, N Sweden $\left(68^{\circ} 25^{\prime} \mathrm{N}\right.$ Lat, $18^{\circ} 20^{\prime} \mathrm{E}$ Long). Coll. 1963 and subm. by Mats Sonesson. Dating is part of current investigations on vegetational dynamics of Torneträsk area. Depths given refer to surface of mire. 
Lu-182. Vassijaure, 195 to $198 \mathrm{~cm}$, peat

Scorpidium peat. Lower limit of peat composed mainly of Scorpidium scorpioides and Calliergon trifarium (slightly humified), just above mineral substratum. Comment: $\mathrm{HCl}$ and $\mathrm{NaOH}$ pretreatment.

Lu-182A. Vassijaure, 195 to $198 \mathrm{~cm}$, humic acid

$8100 \pm 100$

6150 в.c.

$\mathrm{NaOH}$-soluble fraction from material used for Lu-182.

$$
\delta C^{13}=-26.9 \%
$$

Lu-183. Vassijaure, 145 to $146 \mathrm{~cm}$, peat

$5860 \pm 100$

3910 B.C. $\delta C^{13}=-26.4 \%$ o

Scorpidium peat. Upper limit of peat composed mainly of Scorpidium scorpioides and Calliergon trifarium (slightly humified). Comment: $\mathrm{HCl}$ and $\mathrm{NaOH}$ pretreatment.

Lu-183A. Vassijaure, 145 to $146 \mathrm{~cm}$, humic acid

$$
\begin{aligned}
& \mathbf{5 7 4 0} \pm \mathbf{1 0 0} \\
& \mathbf{3 7 9 0} \text { B.C. } \\
& \delta C^{13}=-29.3 \%
\end{aligned}
$$

$\mathrm{NaOH}$-soluble fraction from material used for Lu-183.

Lu-184. Vassijaure, 120 to $121 \mathrm{~cm}$, peat

$$
4630 \pm 100
$$

2680 B.C.

$\delta C^{13}=-26.8 \%$ o

Sphagnum peat. Decreasing Pinus and increasing Betula. Lower limit of peat composed mainly of S. teres (slightly humified). Comment: $\mathrm{HCl}$ and $\mathrm{NaOH}$ pretreatment.

\section{Lu-184A. Vassijaure, 120 to $121 \mathrm{~cm}, \quad 4520 \pm 100$ humic acid

$\mathrm{NaOH}$-soluble fraction from material used for Lu-184.

Lu-185. Vassijaure, 80 to $81 \mathrm{~cm}$, peat

$$
4030 \pm 100
$$

Sphagnum peat. Empiric Picea limit in pollen profile. Upper limit of peat composed mainly of $S$. teres (slightly humified). Comment: $\mathrm{HCl}$ and $\mathrm{NaOH}$ pretreatment.
Lu-185A. Vassijaure, 80 to $81 \mathrm{~cm}$, humic acid
$4050 \pm 100$

$\mathrm{NaOH}$-soluble fraction from material used for Lu-185. 
Lu-186. Vassijaure, 10 to $11 \mathrm{~cm}, \quad 101.9 \pm 0.6 \%$ modern peat $\delta C^{13}=-30.1 \%$

Sphagnum peat. Distinctly decreasing Pinus and increasing Betula. Comment: $\mathrm{HCl}$ and $\mathrm{NaOH}$ pretreatment.

Lu-186A. Vassijaure, 10 to $11 \mathrm{~cm}, \quad 101.2 \pm \underset{\delta C^{13}}{\mathbf{0 . 6} \%}=-28.3 \%$
humic acid

$\mathrm{NaOH}$-soluble fraction from material used for Lu-186.

\section{B. Norway}

Finnmark series, permafrost forms

Continued from Lund I (Radiocarbon, 1968, v. 10, p. 44-45). Peat samples from bottom of polygon furrows from sites in Finnmark, $\mathrm{N}$ Norway. Ref. Svensson (1963). Coll. 1967 and subm. by Harald Svensson, Sci. Res. Council, Stockholm.

Lu-115. Båtsfjord, Finnmark, peat

$$
\delta C^{13}=\begin{array}{r}
<\mathbf{1 8 0} \\
-26.6 \%
\end{array}
$$

Peat from bottom of polygon furrow at Båtsfjord, Finnmark $\left(70^{\circ}\right.$ $35^{\prime} \mathrm{N}$ Lat, $29^{\circ} 40^{\prime} \mathrm{E}$ Long). Comment: $\mathrm{HCl}$ and $\mathrm{NaOH}$ pretreatment; $3 \sigma$ is used for calculation of age limit.

Lu-115A. Båtsfjord, Finnmark, humic acid

$$
\delta C^{13}=-27.9 \%
$$

$\mathrm{NaOH}$-soluble fraction from material used for Lu-115.

\section{Lu-116. Bussesund, Finnmark, peat}

$$
\begin{aligned}
& 4840 \pm 100 \\
& 2890 \text { B.C. } \\
& \delta C^{13}=-28.0 \% \text { }
\end{aligned}
$$

Peat from bottom of polygon furrow at Bussesund, Finnmark $\left(70^{\circ}\right.$ $20^{\prime} \mathrm{N}$ Lat, $31^{\circ} 00^{\prime} \mathrm{E}$ Long). Comment: $\mathrm{HCl}$ and $\mathrm{NaOH}$ pretreatment.

\section{Lu-116A. Bussesund, Finnmark, humic acid \\ $4890 \pm 100$ \\ 2940 B.C.

$$
\begin{aligned}
& \mathbf{2 9 4 0} \text { B.C. } \\
& \delta C^{13}=-27.6 \% \text {. }
\end{aligned}
$$

$\mathrm{NaOH}$-soluble fraction from material used for Lu-116.

II. ARCHAEOLOGIC SAMPLES

\section{Gårdlösa series}

Sweden

Charcoal samples from Gårdlösa No. 3, Smedstorp parish, Scania (55 $34^{\circ} \mathrm{N}$ Lat, $14^{\circ} 08^{\prime} \mathrm{E}$ Long). Coll. 1963 to 1966 and subm. by Berta Stjernquist, Hist. Mus., Univ. of Lund. Several samples from this site have been dated in Uppsala (Radiocarbon, 1965, v. 7, p. 326-327 and Radiocarbon, 1967 , v. 9, p. 465-467). HCl pretreatment of all samples. 
Lu-90. Gårdlösa 3, House VIII

$$
\begin{aligned}
& 1490 \pm 100 \\
& \text { A.D. } 460 \\
& \delta C^{13}=-24.7 \% \text { o }
\end{aligned}
$$

Charcoal from Gårdlösa 3, House VIII, from cultural layer in house foundation. Coll. 1963.

Lu-91. Gårdlösa 3, House XIV

$$
\begin{aligned}
& 1380 \pm 100 \\
& \text { A.D. 570 } \\
& \delta C^{13}=-25.1 \% \text { o }
\end{aligned}
$$

Charcoal from Gårdlösa 3, House XIV, from house foundation, floor level. Coll. 1964.

\section{Lu-92. Gårdlösa 3, House XVII}

$$
\begin{aligned}
& 1680 \pm 100 \\
& \text { A.D. } 270 \\
& \delta C^{13}=-25.2 \%
\end{aligned}
$$

Charcoal from Gårdlösa 3, House XVII, from hearth in house foundation. Coll. 1964.

\section{Lu-93. Gårdlösa 3, House XXIII}

$$
\begin{aligned}
& 1470 \pm 100 \\
& \text { A.D. } 480 \\
& \delta C^{13}=-22.6 \%
\end{aligned}
$$

Charcoal from Gårdlösa 3, House XXIII, from cultural layer in house foundation. Coll. 1965.

\section{Lu-94. Gårdlösa 3, House XXVI}

$$
1260 \pm 100
$$

\section{A.D. 690}

$\delta C^{13}=-24.4 \%$

Charcoal from Gårdlösa 3, House XXVI, from hearth in house foundation. Coll. 1965.

\section{Lu-95. Gårdlösa 3, House XXXII}

$$
1420 \pm 100
$$

\section{A.D. 530}

$$
\delta C^{13}=-26.9 \%
$$

Charcoal from Gårdlösa 3, House XXXII, from cultural layer in house foundation. Coll. 1965.

\section{Lu-96. Gårdlösa 3, House XXXIV}

$$
\begin{aligned}
& 1240 \pm 100 \\
& \text { A.D. } 710 \\
& \delta C^{13}=-25.7 \%
\end{aligned}
$$

Charcoal from Gårdlösa 3, House XXXIV, from cultural layer in house foundation. Coll. 1965.

\section{Lu-97. Gårdlösa 3, House XXXV}

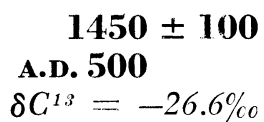

Charcoal from Gårcllösa 3, House $\mathrm{XXXV}$, from hearth in house foundation. Coll. 1965.

\section{Lu-98. Gårdlösa 3, House XXXVIII}

$$
\begin{aligned}
& \mathbf{1 3 7 0} \pm \mathbf{1 0 0} \\
& \text { A.D. 580 } \\
& \delta C^{13}=-26.3 \%
\end{aligned}
$$

Charcoal from Gårdlösa 3, House XXXVIII, from cultural layer in house foundation. Coll. 1965. 


\section{Lu-99. Gårdlösa 3, House XLVIII}

Charcoal from Gårdlösa 3, House XLVIII, from cultural layer in house foundation. Coll. 1964.

\section{Lu-100. Gårdlösa 3, House L}

$1270 \pm 100$

A.D. 680

$\delta C^{13}=-25.1 \%$

Charcoal from Gårdlösa 3, House L, from hearth in house foundation. Coll. 1966.

\section{Hagestad series}

Continued from Lund I (Radiocarbon, 1968, v. 10, p. 48-50). Samples from excavations at Hagestad, Löderup parish, Scania. Coll. 1961 to 1966 and subm. by Märta Strömberg, Hist. Mus., Univ. of Lund. Investigated area described by submitter (Strömberg, 1961, 1963).

\section{Lu-105. Hagestad No. 19², House 1}

$$
\begin{aligned}
& 1280 \pm 100 \\
& \text { A.D. } 670 \\
& \delta C^{13}=-25.7 \% o
\end{aligned}
$$

Charcoal from lower floor level, House 1, at Hagestad No. 19², on field S of No. $19^{3}$ A (55 $24^{\prime}$ N Lat, $14^{\circ} 10^{\prime}$ E Long). Coll. 1961.

\section{Lu-106. Hagestad No. 19², House 4}

Charcoal from hearth, House 4, at Hagestad No. 192, on field $S$ of No. $19^{3} \mathrm{~A}\left(55^{\circ} 24^{\prime} \mathrm{N}\right.$ Lat, $14^{\circ} 10^{\prime} \mathrm{E}$ Long). Coll. 1962.

\section{Lu-107. Hagestad No. 19², House 5}

$$
1180 \pm 100
$$

Charcoal from oven, $\mathrm{N}$ trench, House 5 , at Hagestad No. $19^{2}$, on field $S$ of No. $19^{3}$ A (55 $24^{\prime}$ N Lat, $14^{\circ} 10^{\prime}$ E Long). Coll. 1962. Comment: sample undersized; diluted with "dead" $\mathrm{CO}$, from anthracite coal. Date based on 3 1-day counts.

\section{Lu-108. Hagestad No. 38 $^{6}$, House 1}

$$
\begin{aligned}
& 1150 \pm 100 \\
& \text { A.D. 800 } \\
& \delta C^{13}=-26.3 \%
\end{aligned}
$$

Charcoal from hearth, House 1, at Hagestad No. 38, on field SE of No. $38^{2}\left(55^{\circ} 24^{\prime} \mathrm{N}\right.$ Lat, $14^{\circ} 11^{\prime} \mathrm{E}$ Long). Coll. 1966.

\section{Lu-109. Hagestad No. $38^{6}$, House 3}

$$
\begin{array}{r}
1050 \pm 100 \\
\text { A.D. } 900
\end{array}
$$$$
\delta C^{13}=-26.5 \%
$$

Charcoal from hearth, House 3 , at Hagestad No. $38^{6}$, on field SE of No. $38^{2}$ (55 $24^{\prime}$ N Lat, $14^{\circ} 11^{\prime}$ E Long). Coll. 1966. Comment: sample undersized; diluted. Date based on 3 1-day counts. 
Lu-110. Hagestad No. $33^{4}$ A, Trench 4

Charcoal from hut floor, Trench 4, at Hagestad No. $33^{4}$ A $\left(55^{\circ} 24^{\prime}\right.$ N Lat, $14^{\circ} 11^{\prime}$ E Long). Coll. 1963. Comment: sample undersized; diluted. Date based on 3 1-day counts.

\section{Lu-111. Hagestad No. $6^{3}$}

$$
\begin{array}{r}
1990 \pm 100 \\
40 \text { B.c. } \\
\delta C^{13}=-24.3 \% \text {. }
\end{array}
$$

Charcoal from big hearth, at Hagestad No. $6^{3}$, on field $S$ of coast rd. (55 $23^{\circ} \mathrm{N}$ Lat, $14^{\circ} 09^{\prime}$ E Long). Coll. 1962.

\section{Lu-112. Hagestarl No. $6^{+}$}

$$
\begin{aligned}
& \mathbf{1 8 8 0} \pm 100 \\
& \text { A.D. } 70 \\
& \delta C^{13}=-23.7 \%
\end{aligned}
$$

Charcoal from cultural layer, Square $\mathrm{x}=-15, \mathrm{y}=+20$, at Hagestad No. $6^{4}\left(55^{\circ} 23^{\prime}\right.$ N Lat, 14 $09^{\prime}$ E Long). Coll. 1962.

\section{Lu-113. Hagestad No. $43^{8}$, Grave 3}

$2830 \pm 100$ 880 B.C. $\mathrm{N}$ Lat, $14^{\circ} 08^{\prime}$ E Long). Coll. 1962. Comment: sample undersized; diluted. Date based on 3 1-day counts.

\section{Lu-114. Hagestad No. $19^{3}$ A, Trench 5}

Charcoal from Trench 5 at Hagestad No. $19^{3}$ A $\left(55^{\circ} 24^{\prime} \mathrm{N}\right.$ Lat, $14^{\circ} 10^{\prime}$ E Long). Coll. 1961.

General Comments (M.S.): all dates correspond well with archaeologic results. (S.H.): $\mathrm{HCl}$ pretreatment of all samples.

\section{Bussevik-Senoren series, submarine blockings}

Continued from Lund I (Radiocarbon, 1968, v. 10, p. 50). Wood samples from artificial blockings found in natural channel between Torhamn Peninsula and Senoren I., E Blekinge $\left(56^{\circ} 07^{\prime} \mathrm{N}\right.$ Lat, $15^{\circ} 47^{\prime}$ E Long). Coll. 1967 and subm. by B. E. Berglund, Dept. Quaternary Geol., Univ. of Lund.

\section{Lu-143. Bussevik-Senoren 4}

$$
\begin{aligned}
& 920 \pm 100 \\
& \text { A.D. } 1030 \\
& \delta C^{13}=-25.4^{\prime /} / c 0
\end{aligned}
$$

Wood from oak pile. Sample taken from 10 youngest annual rings of 90-yr old trunk.

\section{Lu-144. Bussevik-Senoren 5}

Wood from oak pile. Sample taken from 10 youngest annual rings of 80-yr old trunk. 


\title{
Lu-145. Bussevik-Senoren 6
}

$1000 \pm 100$ A.D. 950

$\delta C^{13}=-29.2 \%$

Wood from oak pile. Sample taken from 20 youngest annual rings of 130 -yr old trunk.

\section{Lu-146. Bussevik-Senoren 7}

$910 \pm 100$ A.D. 1040

$\delta C^{13}=-27.6 \%$

Wood from aspen pile. Sample taken from 10 youngest annual rings of 40-yr old trunk.

General Comments (B.E.B.): new dates confirm older ones. (S.H.): $\mathrm{HCl}$ pretreatment. All samples charred in nitrogen atmosphere prior to burning.

\section{Lu-103. Gudahagen, Näsum, human bone}

$$
\begin{aligned}
& 1030 \pm 100 \\
& \text { A.D. 920 } \\
& \delta C^{13}=-19.4 \%
\end{aligned}
$$

Collagen from human femur from Gudahagen, Näsum parish, Scania $\left(56^{\circ} 10^{\prime} \mathrm{N} \mathrm{Lat}, 14^{\circ} 30^{\prime} \mathrm{E}\right.$ Long). Whole skeleton was found in stone cist. Cist was covered by ca. $1 / 2 \mathrm{~m}$ of gravel and stones. Coll. 1967 and subm. by Claes Wahlöö, Hist. Mus., Univ. of Lund. Comments (C.W.): expected age 1000 to $1500 \mathrm{yr}$. (S.H.): collagen fraction treated with cold $0.5 \%$ $\mathrm{NaOH}$ solution for extraction of humic acid.

Date lists:

\section{REFERFNCIS}

\author{
Lund I Håkansson, 1968 \\ Uppsala $V \quad$ Olsson and Plyanuj, 1965 \\ Uppsala VII Olsson, Stenberg, and Göksu, 1967
}

Alin, Johan and Sandegren, Ragnar, 1947, Dösebackaplatån: Sreriges Geol. Unders., ser. C., no. 482, p. 3-40.

Antevs, E., 1917, Post-glacial marine shell-beds in Bohuslän: Geol. För. Stockholm Förh., v. 39, p. 247-425.

Berglund, B. E., 1966, Late-Quaternary vegetation in eastern Blekinge, southeastern Sweden. II. Post-Glacial time: Op. Bot. A Soc. Bot. Lundensi, v. 12:2, p. 1-190.

De Geer, G., 1910, Quaternary sea-bottoms in Western Sweden: Geol. För. Stockholm Förh., v. 32, p. $1139-1195$

Digerfeldt, Gunnar, 1966, Utvecklingshistoriska och limnologiska observationer i Ranviken av sjön Immeln (summary in English): Bot. Notiser (Lund), v. 119, fasc.
2, p. 216-232.

Hâkansson, Sören, 1968, University of Lund radiocarbon dates I: Radiocarbon, v. 10, p. $36-54$.

Hillefors, Ake, 1961, On wind-eroded boulders and stones in horizons in the Dösebacka buildings, near Gothenburg: Gothia, Medd. från Geog. För. i Göteborg, no. 9, p. 73-93.

im Kattegat, Die Würmzeitlichen Eisbewegungen und der Verlauf der Deglaziation

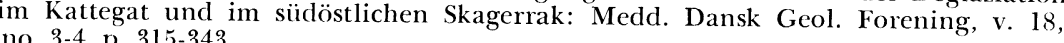

Kurck, C., 1922, Faunan och floran i några sydskånska, hittills obeskrivna kalktuffer: Arkiv f. kemi, mineralogi och geol., v. 8, no. 15, p. 1-70.

Nilsson, Tage, 1927, En skalbanksstudie i Bohuslän: Geol. För. Stockholm Förh., v. 49 , p. $321-348$

1964, Standardpollendiagramme und $\mathrm{C}^{14}$-datierungen aus dem Ageröds p. $1-52$. 
Olsson, Ingrid U. and Piyanuj, Piya, 1965, Uppsala natural radiocarbon measurements V: Radiocarbon, v. 7, p. 315-330.

Olsson, Ingrid U., Stenberg, Allan, and Göksu, Yeter, 1967, Uppsala natural radiocarbon measurements VII: Radiocarbon, v. 9, p. 454-470.

Sonesson, Mats, 1968, Pollen zones at Abisko, Torne Lappmark, Sweden: Bot. Notiser (Lund), v. 121, fasc. 4, p. 491-500.

Strömberg, Märta, 1961, Eine siedlungsgeschichtliche Untersuchung in Hagestad, Südost-Schonen: Medd. från Lunds Univ. Hist. Mus. 1961, p. 123-154.

1963, Handelsstråk och vikingabygd i sydöstra Skåne: Ale, Hist. Tidskr. för Skåneland, no. 3, 1963, p. 1-25.

Svensson. Harald, 1963, Tundra polygons; photographic interpretation and field studies in North-Norwegian polygon areas: Norges Geol. Unders., ser. C, no. 598, p. 1-29.

Welinder, Stig, 1968, Supramarine Litorina layers at Segevången, Malmö: Geol. För. Stockholm Förh., v. 90, p. 126-128. 